\title{
Search for neutral and charged BSM Higgs Bosons with the ATLAS detector
}

\author{
Pawel Brückman de Renstrom ${ }^{1, a}$ on behalf of the ATLAS Collaboration \\ ${ }^{1}$ Institute of Nuclear Physics, Polish Academy of Sciences, ul. Radzikowskiego 152, 31-342 Cracow, Poland
}

\begin{abstract}
Several theories beyond the Standard Model, like the EWS or 2HDM models, predict the existence of high mass neutral or charged Higgs particles. The latest ATLAS results on searches for these particles will be briefly reviewed.
\end{abstract}

\section{Introduction}

The discovery of the fundamental scalar in 2012 [1, 2], together with no observation of light supersymmetric particles, triggered an understandable interest in exploring the scalar sector in search for evidence of beyond Standard Model (BSM) phenomena. So far the discovered particle manifests properties expected from the Higgs particle of the Standard Model (SM). It's mass has been precisely measured to be $125.0 \pm 0.3 \mathrm{GeV}[3,4]$ and cross-section times branching fraction to observed final states are consistent with that predicted by the SM. Consequently, couplings to SM particles appear proportional to their mass for fermions and to mass squared for bosons, in agreement with the SM prediction within the uncertainties [5].

The apparent consistency with the SM does not exclude new physics scenarios, though. The basic question is whether the $125 \mathrm{GeV}$ state is the only fundamental scalar or maybe the Higgs sector is extended. Consequently, is it responsible for the entire mass of all particles? Finally, is it fundamental? In order to address these questions one defines models of interest to be tested. Most commonly considered are additional electroweak singlet (EWS) [6], Two Higgs Doublet Model (2HDM) [7], various Higgs triplet models [8-10] or composite Higgs models [11]. All of them allow for SM-like phenomenology of the lightest scalar, with different coupling modifications.

The investigation naturally splits in two categories. First one explores the properties of the $125 \mathrm{GeV}$ scalar measuring the cross-sections of various production modes, decay widths to different final states, spin and parity as well as searching for exotic decays of the Higgs, e.g. invisible. The second conducts explicit searches for BSM scalars.

This report focusses on the latter category and reports on the recent ATLAS [12] results of searches for heavier members of the scalar sector. Unless otherwise stated, shown results are based on the full $2015+2016$ dataset ( $36 \mathrm{fb}^{-1}$ of $13 \mathrm{TeV} p p$ collisions). Presented analyses often report interpretations within multiple models, including heavy vectors or spin-two heavy objects. Only results targeting heavy scalar scenarios are discussed in the following.

\footnotetext{
ae-mail: pawel.bruckman.de.renstrom@cern.ch
} 
The report is divided into three main sections discussing searches for heavy neutral Higgs decaying to bosons, heavy neutral Higgs decaying to fermions and searches for a charged or doubly-charged Higgs.

\section{Heavy neutral Higgs decaying to bosons}

A search for $\gamma \gamma$ resonance using the full $36.7 \mathrm{fb}^{-1}$ of data collected in 2015 and 2016 was eagerly awaited owing to the mild excess around $750 \mathrm{GeV}$ reported from 2015 data by both ATLAS and CMS collaborations $[13,14]$. The search was performed in a wide range of masses: $200 \mathrm{GeV}<m_{\gamma \gamma}<2700 \mathrm{GeV}$ and for a narrow and wide width hypothesis: $\Gamma_{\mathrm{H}} / m_{\mathrm{H}}=[0 \%-10 \%][15]$. The main requirement are two energetic photons isolated using both calorimeter and tracking detector information ${ }^{1}$. The analysis was separately optimised for spin- 0 and spin- 2 hypotheses. Scalar hypothesis leads to more centrally emitted photons. $90 \%$ of the background consisted of irreducible di-photon production, the rest being $\gamma$ jet and dijet. The background modelling was based on a smooth functional form, with the signal and background yields determined simultaneously by the fit. Figure 1 shows the $m_{\gamma \gamma}$ distribution obtained for the spin-0 hypothesis selection together with residuals to the fitted background shape on the left and the limit on cross-section times branching fraction to $\gamma \gamma$ resulting from the maximum-likelihood fits to signal-plus-background models on the right. The largest
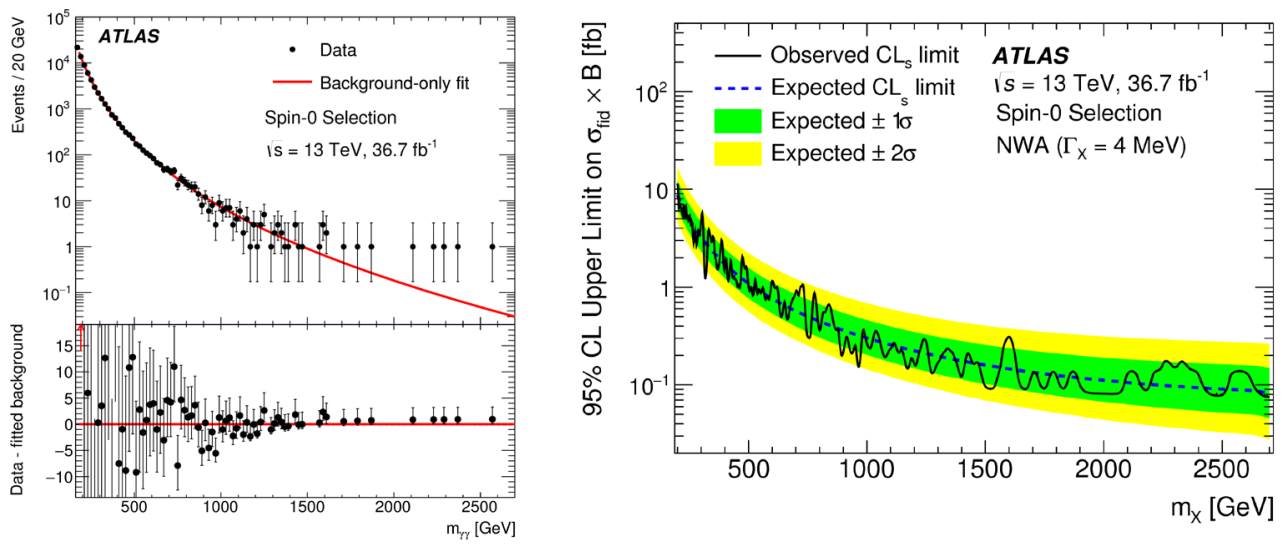

Figure 1. Principal results of the $H \rightarrow \gamma \gamma$ search. Shown are the $m_{\gamma \gamma}$ distribution obtained for together with residuals to the fitted background shape (left) and the 95\% CL upper limit on the cross-section times branching fraction to $\gamma \gamma$ resulting from the maximum-likelihood fits to the signal-plus-background models (right). The observed (solid line) and expected (dashed line) upper limits are shown [15].

local excess of $2.6 \sigma$ around $730 \mathrm{GeV}$ for narrow width is driven by 2015 data and reduces to less than one $\sigma$ global significance (when the look elsewhere effect is taken into account) ${ }^{2}$.

\footnotetext{
${ }^{1}$ The scalar sum of the transverse energy of clusters within a cone of size $\Delta R=\sqrt{(\Delta \eta)^{2}+(\Delta \phi)^{2}}=0.4$ around the photon candidate, excluding the photon energy deposits and correcting for pile-up and underlying-event contributions, is required to be below $0.022 E_{\mathrm{T}}+2.45 \mathrm{GeV}$, where $E_{\mathrm{T}}$ is the transverse energy of the photon candidate. The sum of the transverse momentum $\left(p_{\mathrm{T}}\right)$ of tracks within $\Delta R=0.2$ of the photon candidate is required to be below $0.05 E_{\mathrm{T}}$.

${ }^{2}$ Less than the median largest deviation in background-only pseudo-experiments.
} 
A related search for a resonance in the $Z \gamma$ final state was also performed [16]. Similarity comes from the same Feynman diagrams responsible for the Higgs decay, hence a similar expected crosssection. A photon, fulfilling similar requirements as in the previous search, was paired with a $Z$ candidate decaying to either a pair of electrons or muons. An $e(\mu)$ had to be matched to the fired lepton trigger and satisfy $p_{\mathrm{T}}>18(24) \mathrm{GeV}$. The main backgrounds arise from irreducible non-resonant $Z \gamma$ production and reducible $Z+$ jets and are estimated from a fit of an analytical model to data. The functional form is taken from Monte Carlo simulation while the relative contribution of the two main backgrounds is estimated from a dedicated control region. The result of the background-only fit of the model to data in the example $\mu \mu$ signal region is shown in the left panel of Figure 2 . The largest
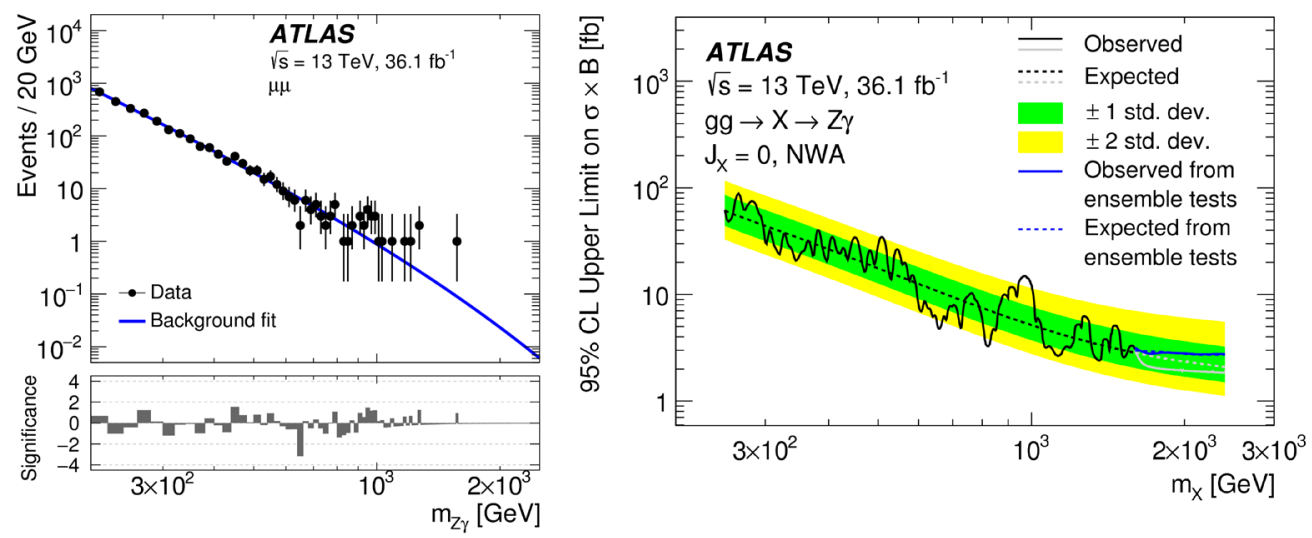

Figure 2. Principal results of the $H \rightarrow Z \gamma$ search. Shown are the background-only fit to the $m_{Z \gamma}$ distribution together with residuals to the fitted background shape (left) and the 95\% CL upper limit on the cross-section times branching fraction to $Z \gamma$ resulting from the maximum-likelihood fits to the signal-plus-basckground models (right). The observed (solid line) and expected (dashed line) upper limits are shown assuming production via gluon-gluon fusion and using the narrow width approximation [16].

local excess of $2.7 \sigma$ around $960 \mathrm{GeV}$ has a negligible $(0.8 \sigma)$ global significance.

The decay to a pair of $Z$ bosons is one of the principal search channels, notably the golden one with four charged leptons in the final state. The search for heavy scalar was performed in all possible combinations of $Z$ decays except for the fully hadronic and fully invisible ones [17-19]. In the fourlepton search two same-flavour opposite sign lepton pairs were required. All leptons had to satisfy tracking and calorimetric isolation. The signal was searched for in the invariant mass spectrum of four leptons. In this channel background is dominated by the non-resonant $Z Z$ production and is estimated from the simulation. The interference between the resonant signal and the background was properly accounted for. In the search for $\ell \ell v v$ final state a single pair of same-flavour opposite sign isolated leptons was combined with at least $120 \mathrm{GeV}$ of missing transverse energy $\left(E_{\mathrm{T}}^{\mathrm{miss}}\right)$. Additionally, the lepton pair and the missing energy were required to be approximately back-to-back in the azimuthal angle. Here, the background arose from the non-resonant production of either $Z Z(\sim 60 \%)$ or $Z W$ $(\sim 30 \%)$ and was also estimated from the simulation. The transverse mass of the leptonically decaying reconstructed $Z$ and $E_{\mathrm{T}}^{\text {miss }}$ was used as the discriminant variable. In both channels the search was done separately in the gluon-gluon fusion ( $\mathrm{ggF}$ ) and vector-boson fusion (VBF) enriched categories. The latter was tagged by the presence of two hadronic jets with a large invariant mass and large rapidity 
gap. The $95 \% \mathrm{CL}$ upper limit on the cross-section times branching fraction for the $4 \ell$ and $\ell \ell v v$ channels and their combination are shown in Figure 3. The limits for the ggF hypothesis (left) and the VBF hypothesis (right) are shown separately. The limit is dominated by the $4 \ell$ channel in the low
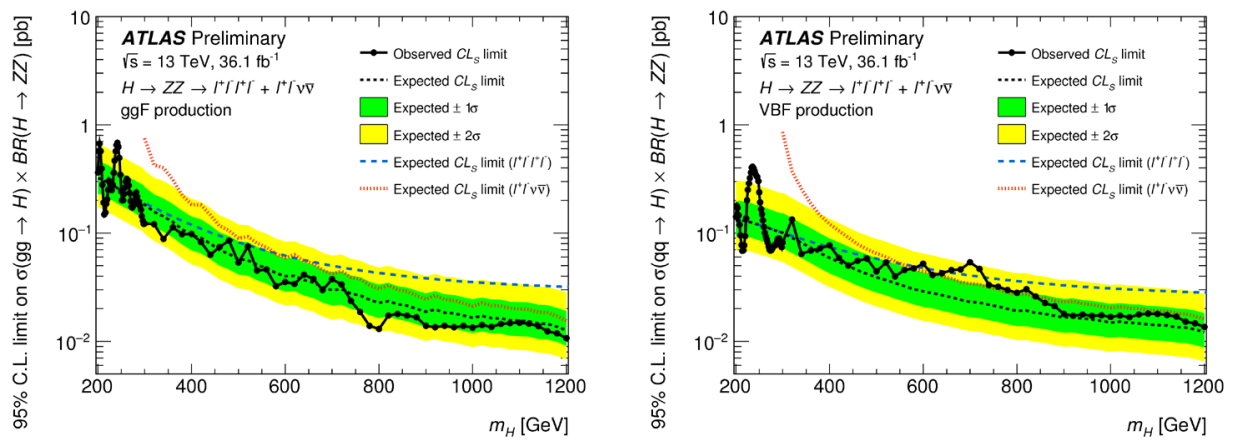

Figure 3. 95\% CL upper limit on $\sigma(g g \rightarrow H) \times \mathrm{BR}(H \rightarrow \mathrm{ZZ})$ for the $g g F$ hypothesis (left) and the VBF hypothesis (right) and the narrow width approximation. Expected limit sensitivity for the $4 \ell$ and $\ell \ell v v$ channels and their combination are shown separately [17].

mass region due to the better mass resolution while for high masses larger branching of $Z \rightarrow v v$ gains. The $4 \ell$ shows 3.6 (2.2) $\sigma$ local (global) excesses at around $240 \mathrm{GeV}$ and $700 \mathrm{GeV}$. The lower mass one is not covered by the $\ell \ell v v$ search which starts only at $300 \mathrm{GeV}$. Significance of the $700 \mathrm{GeV}$ excess reduces to $2.0(1.0) \sigma$ local (global) when combined with the $\ell \ell v v$ channel. An example interpretation of the limits in the 2HDM Type-2 model is shown in Figure 4. The two plots give the complementary set of exclusions in $[\cos (\beta-\alpha), \tan \beta]$ and $\left[m_{H}, \tan \beta\right]$ planes.
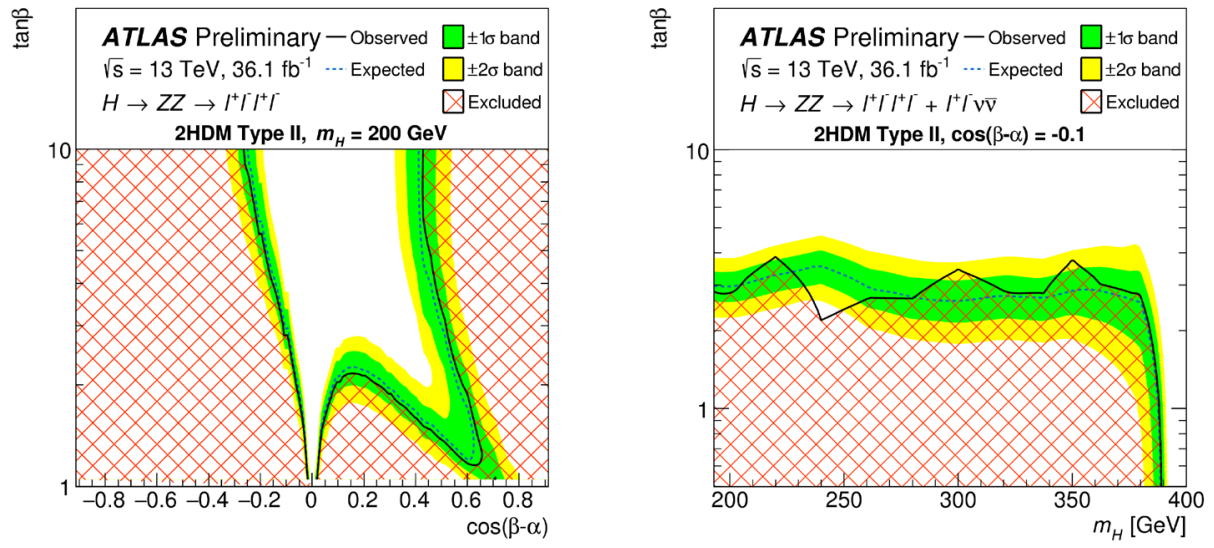

Figure 4. The exclusion contours in the 2HDM Type- 2 model. Shown are exclusion for $m_{H}=200 \mathrm{GeV}$ as a function of $\cos (\beta-\alpha)$ and $\tan \beta$ parameters (left) and exclusion for $\cos (\beta-\alpha)=-0.1$ as a function of $m_{H}$ and $\tan \beta$. The hatched area shows the observed exclusion [17]. 
Two searches for a heavy $H \rightarrow Z Z \rightarrow \ell \ell q q / v v q q$ and CP-odd $A \rightarrow Z h \rightarrow \ell \ell b b / v v b b$ were performed owing to improved dijet mass resolution which used both the calorimeter and tracking information. The searches looked for either a pair of same-flavour opposite charge isolated leptons or large missing transverse energy together with either a merged fat jet $(R=1.0)$ or a pair of narrow jets $(R=0.4)$. The merged and resolved candidates were looked for sequentially, covering a large range of heavy resonance mass hypothesis. In the $H \rightarrow Z Z$ search, the candidates were categorised as ggF or VBF in the presence of additional two forward jets with high $\Delta \eta_{j j}$ and high $m_{j j}$. The search for $A \rightarrow Z Z$ additionally required the jets to be identified as originating from a $b$-quark ( $b$-tagged) and were subsequently categorised as $b b A$ associated production in the presence of more than two $b$-tagged jets in the event. Main backgrounds to the analyses originated from $Z+$ jets and $W+$ jets and were modelled by the Monte Carlo with the normalisation taken from data in control regions. Limits on cross-section times branching fraction were extracted from maximum likelihood fits to either $m(\ell \ell j j)$ or $m_{\mathrm{T}}\left(E_{\mathrm{T}}^{\mathrm{miss}} j j\right)$. Figure 5 shows the obtained limits on $\sigma(g g \rightarrow H) \times \mathrm{BR}(H \rightarrow Z Z)$ (left) and $\sigma(\mathrm{VBF} q q \rightarrow H) \times \mathrm{BR}(H \rightarrow Z Z)$ (right). No significant excess was observed. A local
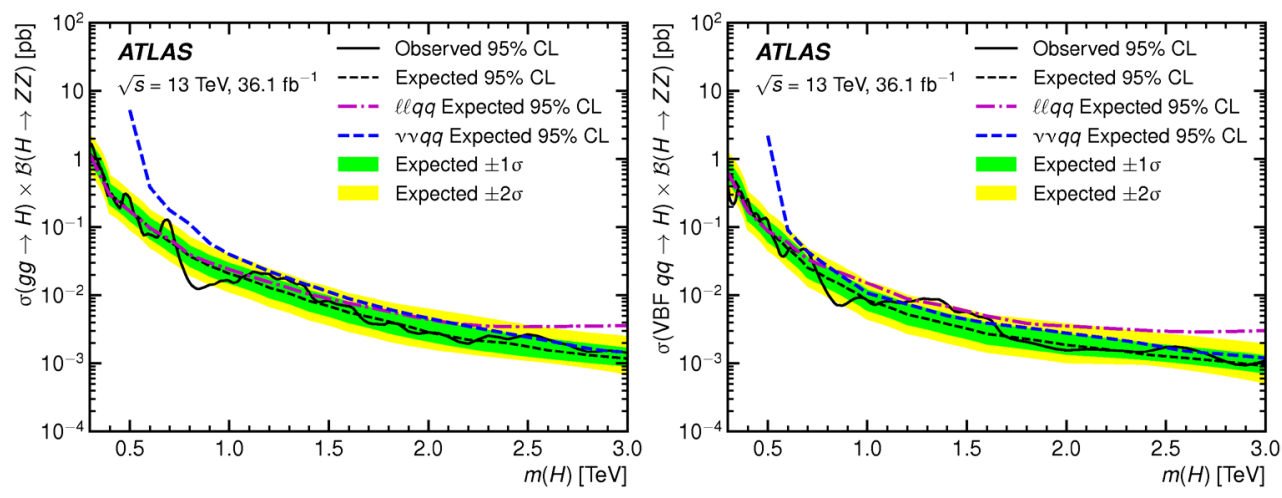

Figure 5. 95\% CL upper limit on $\sigma(g g \rightarrow H) \times \mathrm{BR}(H \rightarrow$ ZZ) (left) and $\sigma(\mathrm{VBF} q q \rightarrow H) \times \mathrm{BR}(H \rightarrow Z Z)$ (right) for the narrow width approximation. Expected limit sensitivity for the $\ell \ell j j$ and $v v j j$ channels and their combination are shown separately [18].

deficit at around $800 \mathrm{GeV}$ corresponds to barely $1.9 \sigma$ globally. Limits resulting from the search for $A \rightarrow Z h \rightarrow \ell \ell b b / v v b b$ are shown in Figure 6 for the gluon fusion production (left) and $b$-associated production (right). The mild excess observed around $440 \mathrm{GeV}$ is driven mainly by the di-muon channel in the resolved category with $\geq 3 b$-tagged jets and amounts to the local (global) significance of $3.6 \sigma(2.4 \sigma)$.

A complementary search for a heavy neutral $H \rightarrow W W \rightarrow \ell v q q$ [20] was also performed. The searched signature consisted of an isolated lepton, $E_{\mathrm{T}}^{\text {miss }}$ and either a merged fat jet (boosted) or a pair of narrow jets (resolved). The analysis additionally distinguished VBF production with two forward jets with high $\Delta \eta_{j j}$ and high $m_{j j}$. Majority of the background originated from $W+$ jets with $t \bar{t}$ being the second important one. Both were modelled by the simulation with the free normalisation in the simultaneous fit to signal region and two control regions enriched in either $W+$ jets or $t \bar{t}$ events. The final discriminating variable was the invariant mass of the $W W$ system, with the semileptonic kinematics obtained from the $m_{W}$ constraint on the $\ell v$ system. The narrow width approximation signal hypothesis in the wide mass range of $300 \mathrm{GeV}<m_{H}<3000 \mathrm{GeV}$ was tested separately for the Drell-Yan ( $g g$ and $q q$ fusion) and VBF production with the profile-likelihood-ratio fit. Results are shown in Figure 7. No significant deviation from the SM background was observed. 

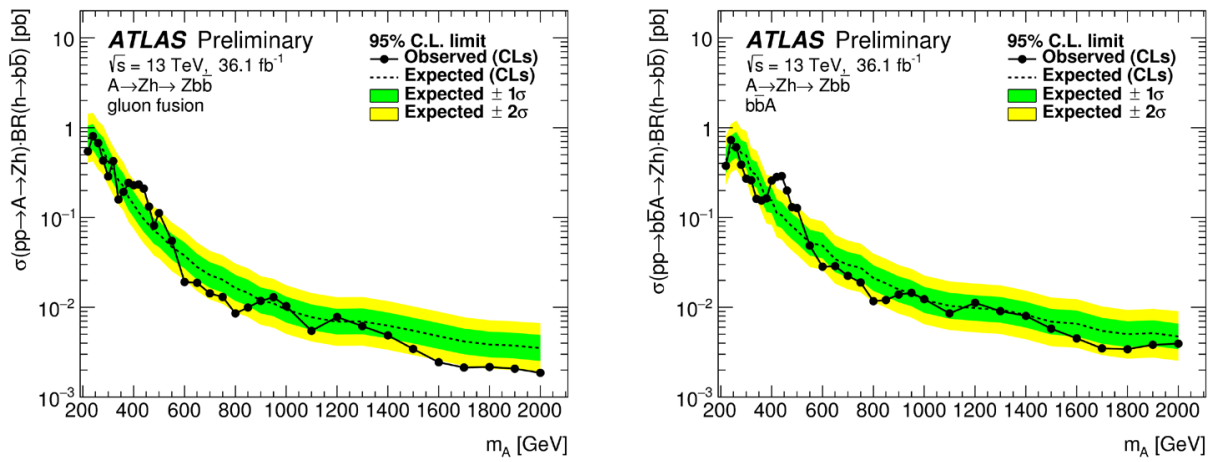

Figure 6. 95\% CL upper limit on $\sigma(g g \rightarrow A \rightarrow Z h) \times \mathrm{BR}(h \rightarrow b \bar{b})$ (left) and $\sigma(p p \rightarrow b \bar{b} H \rightarrow b \bar{b} Z h) \times \mathrm{BR}(h \rightarrow$ $b \bar{b})$ (right). Limits combine both the 0-lepton and 2-lepton channels [19].
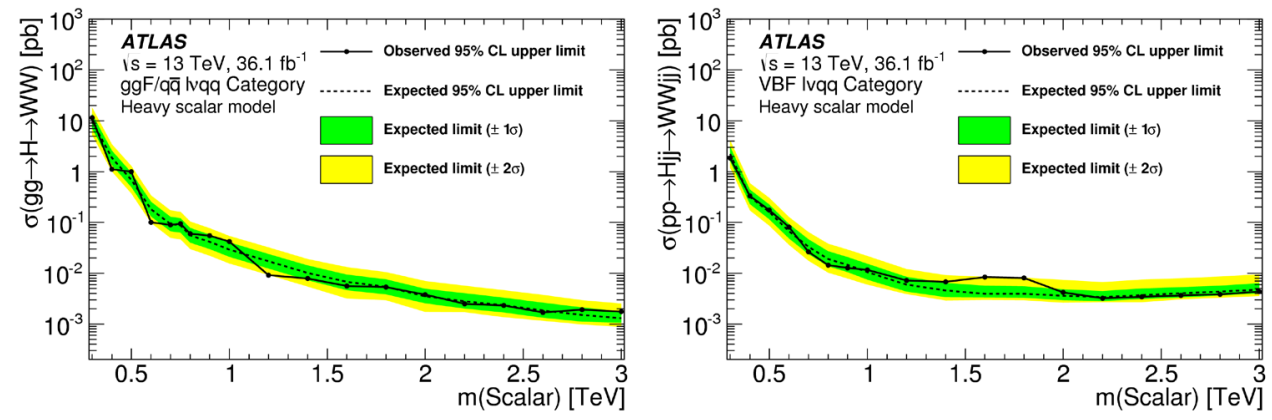

Figure 7. 95\% CL upper limit on $\sigma(p p \rightarrow H \rightarrow W W)$ (left) and $\sigma(p p \rightarrow H j j \rightarrow W W j j)$ (right) [20].

\section{Heavy neutral Higgs decaying to fermions}

A heavy scalar was looked for in its fermionic decay to a pair of $\tau$ leptons in the mass range $200 \mathrm{GeV}<$ $m_{\tau \tau}<2250 \mathrm{GeV}$ [21]. Two search channels were used. In the $\tau_{\text {lep }} \tau_{\text {had }}$ a reconstructed lepton which fired the trigger was paired with an oppositely charged hadronically decaying $\tau$ candidates (1 or 3prong). The $\tau_{\text {had }} \tau_{\text {had }}$ channel required two oppositely charged hadronic $\tau$ candidates with transverse momentum of at least $65 \mathrm{GeV}$ each, one associated with the fired tau trigger. The $b$-tag and $b$-veto categories were defined to distinguish between the ggF and $b$-associated production. The dominating background consisted of events where jets were misidentified as $\tau_{\text {had }}$ candidates (fake taus) in $W+$ jets and $t \bar{t}$ or in multi-jet events for $\tau_{\text {lep }} \tau_{\text {had }}$ and $\tau_{\text {had }} \tau_{\text {had }}$ channels, respectively. It was estimated using, so called, Fake Factor method whereby the ratio of identified fake tau candidates to $\tau$ candidates failing the identification criteria $(F F)$ is extracted from data in a control region. Subsequently, the fake tau contribution in the signal region is modelled by the $\tau$ candidates failing tau identification scaled by the $F F$. In both $\tau_{\text {lep }} \tau_{\text {had }}$ and $\tau_{\text {had }} \tau_{\text {had }}$ channels the total transverse mass of the two tau candidates and the $E_{\mathrm{T}}^{\text {miss }}$ were used in the maximum-likelihood fit. Example result for the ggF category together with the exclusion contour on the $\left[m_{A}, \tan \beta\right]$ plane for the hMSSM [22] scenario are shown in Figure 8. The limits were much improved w.r.t. the ones obtained with 2015 data, yet no significant deviation from limits expected from SM physics alone were observed. 

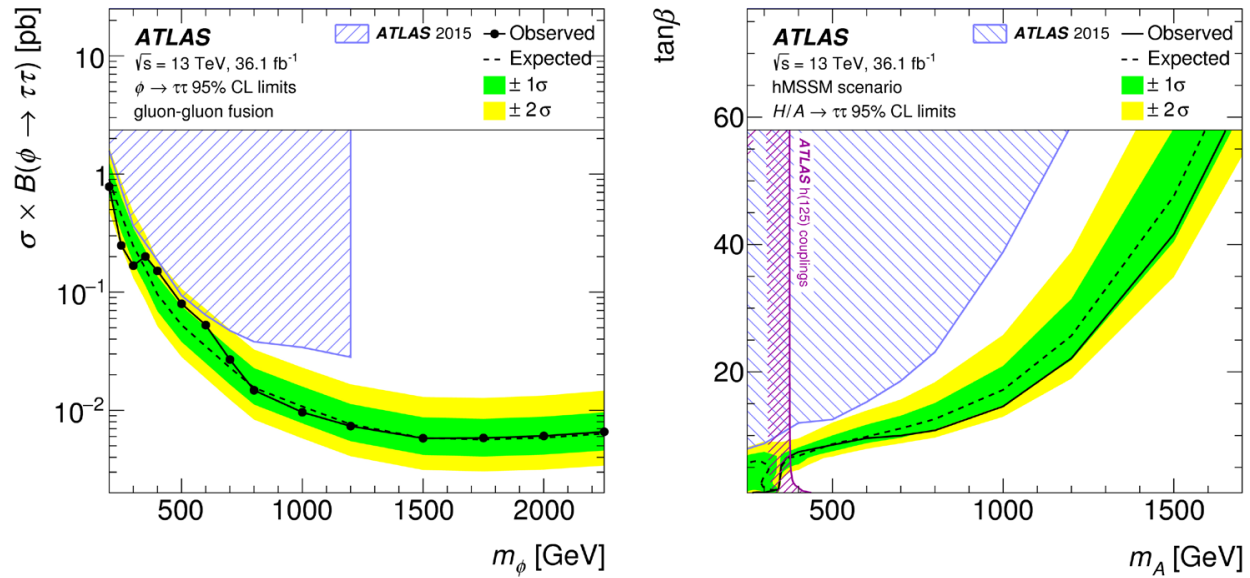

Figure 8. 95\% CL upper limit on $\sigma \times \mathrm{BR}(H \rightarrow \tau \tau)$ for the gluon-gluon fusion production (left) and the $95 \%$ exclusion contour on the $\left[m_{A}, \tan \beta\right]$ plane for the hMSSM scenario (right) [21]. The hMSSM exclusion stems from the combination of ggF and $b$-associated production limits. The excluded regions from the 2015 ATLAS search [23] are depicted by the blue hatched areas.

\section{Search for the charged and doubly-charged Higgs}

A charged scalar of the electroweak symmetry breaking sector would spectacularly sign physics beyond SM. It's presence is mandatory with more than one Higgs doublet, notably within Supersymmetry [24]. Within MSSM [25], the decay $H^{+} \rightarrow \tau v$ saturates the charged Higgs branching for $m_{H}<m_{t}$ and remains significant in large range of masses for high $\tan \beta$. For low $\tan \beta$, though, $H^{+} \rightarrow t b$ dominates for $m_{H}$ above the top mass.

A search for heavy charged Higgs decaying to $\tau v$ for $m_{H}>200 \mathrm{GeV}$ was performed on $14.7 \mathrm{fb}^{-1}$ of data collected in 2015 and first half of 2016 [26]. $H^{+}$production was assumed in association with a top quark. In $E_{\mathrm{T}}^{\text {miss }}$-triggered events a $\tau_{\text {had }}$ candidate, $E_{\mathrm{T}}^{\text {miss }}$ and $\geq 3$ jets with at least one $b$-tagged were required. Dominating backgrounds with true taus were $t \bar{t}, W+$ jets, while multi-jet event gave rise to background with jets faking taus. Fake tau background was modelled using the fake factor method, with $F F$ extracted in the QCD control region defined by a $b$-tag veto and low $E_{\mathrm{T}}^{\mathrm{miss}}$. The limit on $\sigma\left(p p \rightarrow[b] t H^{+}\right) \times \mathrm{BR}\left(H^{+} \rightarrow \tau v\right)$ was based on profile likelihood fits to the transverse mass of the $\left(\tau, E_{\mathrm{T}}^{\mathrm{miss}}\right)$ system and is shown in Figure 9 (left). No significant excess was observed which was converted into exclusion in a number of benchmark models. An example of exclusion in the $\left[m_{H^{+}}, \tan \beta\right]$ plane of the $m_{h}^{\bmod -}$ scenario of the MSSM [27] is shown in Figure 9 (right).

A complementary search for the $t b$ decays of $H^{+}$was performed on the same dataset $\left(14.7 \mathrm{fb}^{-1}\right)$ for masses ranging from $300 \mathrm{GeV}$ to $1 \mathrm{TeV}$ [29]. The assumed top-associated production leads to six jets in the final state out of which four originate from $b$-quarks while one of the top quarks is required to decay semileptonically. Events were selected requiring an isolated lepton associated with a fired leptonic trigger, at least four jets, and at least two $b$-tagged. A veto on reconstructed hadronic $\tau$ candidates assured orthogonality with the $\tau v$ search. Background stemmed mostly from $t \bar{t}+b$-jets events and was modelled from Monte Carlo with the normalisation free in the fit. Several signal regions (SR) and control regions (CR) were defined based on the number of reconstructed jets and number of $b$-tagged ones. Signal regions were defined for $\geq 5$ jets and $\geq 3 b$-tags. Control regions contained fewer number of jets or $b$-tagged jets. The profile likelihood fit was performed simultaneously to the 

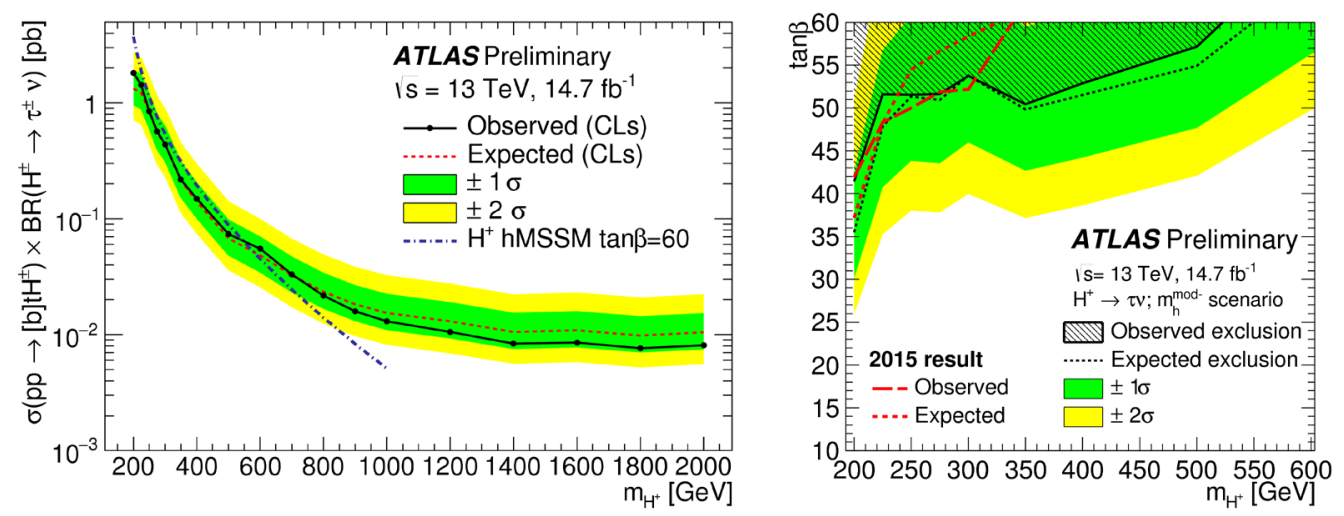

Figure 9. 95\% CL upper limit on $\sigma\left(p p \rightarrow[b] t H^{+}\right) \times \mathrm{BR}\left(H^{+} \rightarrow \tau v\right)$ (left) and the 95\% exclusion contour in the $\left[m_{H^{+}}, \tan \beta\right]$ plane of the $m_{h}^{\text {mod- }}$ scenario (right) [26]. The expected and observed exclusions obtained with the 2015 ATLAS search [28] are overlaid with red lines.

Boosted Decision Trees (BDT) discriminant in the SR and the scalar sum of the $p_{\mathrm{T}}$ of the selected jets $\left(H_{\mathrm{T}}^{\mathrm{h} a d}\right)$ in the $\mathrm{CR}$. The resulting limit on $\sigma\left(p p \rightarrow t b H^{+}\right) \times \mathrm{BR}\left(H^{+} \rightarrow t b\right)$ is shown in Figure 10 (left). No significant excess above the expected SM background was found. An exclusion in the $\left[m_{H^{+}}, \tan \beta\right]$
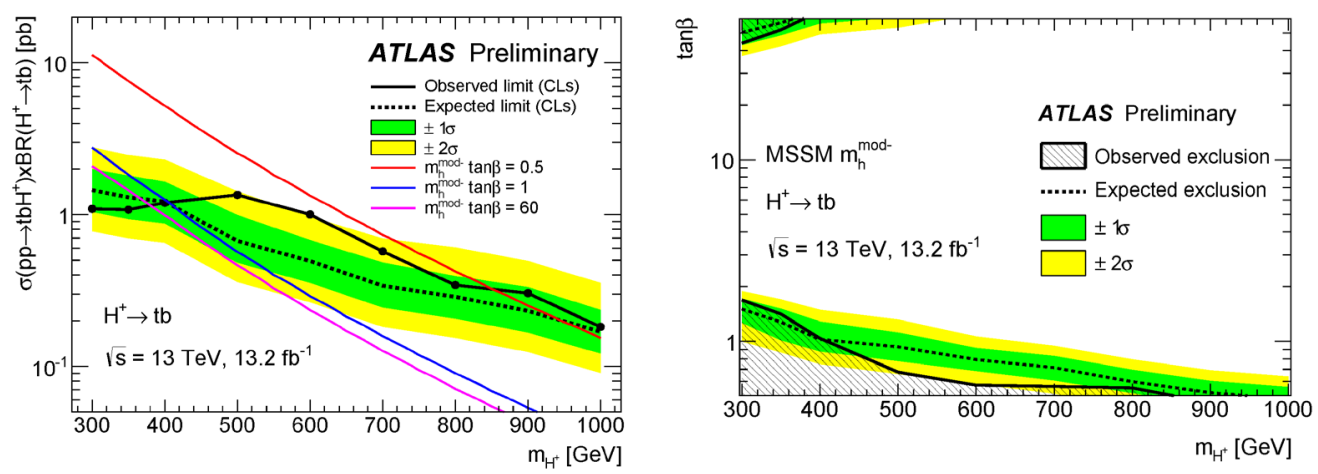

Figure 10. 95\% CL upper limit on $\sigma\left(p p \rightarrow t b H^{+}\right) \times \mathrm{BR}\left(H^{+} \rightarrow t b\right)$ (left) and the $95 \%$ exclusion contour in the $\left[m_{H^{+}}, \tan \beta\right]$ plane of the $m_{h}^{\text {mod- }}$ scenario (right) [29].

plane of the $m_{h}^{\text {mod- }}$ scenario of the MSSM is shown in Figure 10 (right).

BSM scenarios involving scalar triplets lead to heavy-neutral, charged and doubly-charged Higgs bosons $\left(H^{++}\right)$. The latter are expected to be predominantly pair-produced in the Drell-Yan process. The Left-Right Symmetric Model (LRSM) [8] predicts the $H^{++}$decays to be saturated by the $l^{+} l^{+}$ channel for low values of the vacuum expectation value of the triplet $\left(v_{\Delta}\right)$ while for high $v_{\Delta}$ values the decays are completely dominated by the $W^{+} W^{+}$channel.

Doubly charged Higgs in its decays to two same-sign leptons was searched for with $36.1 \mathrm{fb}^{-1}$ of 2015+2016 data [30]. The Drell-Yan pair production results in two same-sign pairs of leptons. The search was done in 2, 3 and 4-lepton categories depending on the number of reconstructed isolated 
leptons. In the firs two categories, the invariant mass of the same-sign lepton pair was used as the discriminating variable. The 4-lepton category used the mean of the invariant mass of the two samesign lepton pairs. Background containing true isolated leptons arose mostly from di-boson and $t \bar{t}$ events and was modelled from Monte Carlo with the charge misidentification rate measured on data. Fake lepton background was estimated using the fake factor method. Figure 11 left shows the 95\% CL upper limit on $\sigma\left(p p \rightarrow H^{ \pm \pm} H^{\mp \mp}\right)$ for the working point which assumes $100 \%$ branching to a $\mu \mu$ pair. Similar limits were obtained for other assumed branchings. In all cases, branching to final states
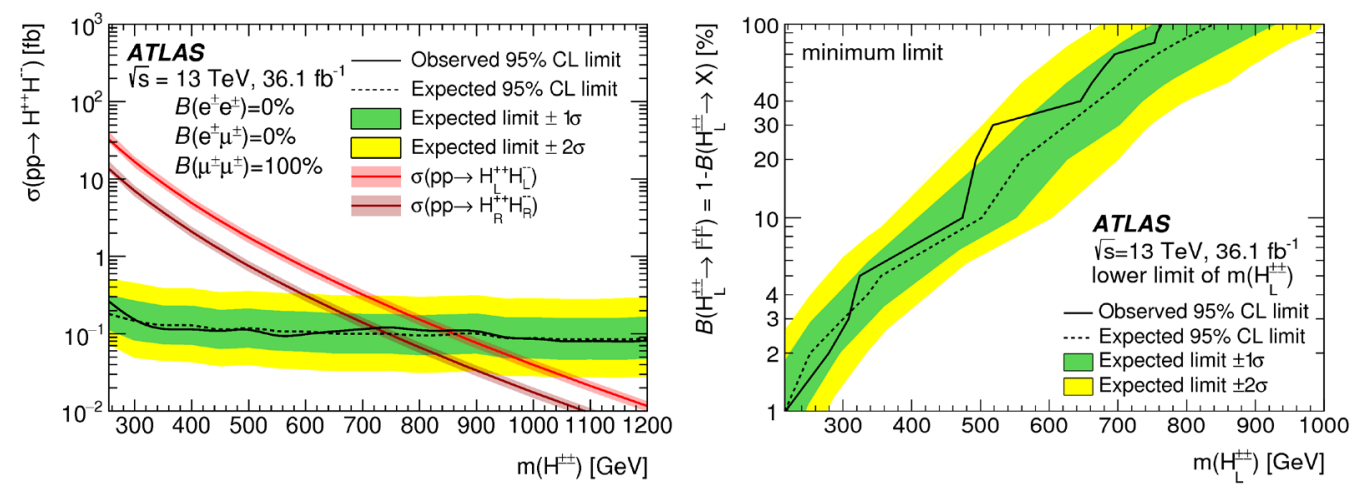

Figure 11. 95\% CL upper limit on $\sigma\left(p p \rightarrow H^{ \pm \pm} H^{\mp \mp}\right)$ for an example working point which assumes $100 \%$ branching to a $\mu \mu$ pair (left) and the $95 \% \mathrm{CL}$ limit on $H_{L}^{ \pm \pm}$mass as a function of branching ratio $\operatorname{Br}\left(H_{L}^{ \pm \pm} \rightarrow l^{ \pm} l^{ \pm}\right)$, where $l$ stands for either $e$ or $\mu$ (right) [30].

other than a lepton pair was assumed to be negligible. The left panel of Figure 11 gives the 95\% CL limit on $H_{L}^{ \pm \pm}$mass as a function of branching ratio $\operatorname{Br}\left(H_{L}^{ \pm \pm} \rightarrow l^{ \pm} l^{ \pm}\right)$, where $l$ stands for either $e$ or $\mu$. $H_{L}^{ \pm \pm}$in the LRSM is the $H^{ \pm \pm}$state coupling to the left-handed fermions and has a few times larger predicted production cross-section than its right-handed counterpart $\left(H_{R}^{ \pm \pm}\right)[31]$.

\section{Summary}

ATLAS conducted a wide variety of searches for BSM heavy scalar particles (CP-even and CP-odd, singly and doubly charged Higgses). Majority of presented results are based on the full 2015+2016 dataset. Remaining ones are being updated and should become public soon. No evidence for BSM phenomena in the scalar sector has been observed so far. Analyses managed to place exclusion limits on various BSM scenarios, notably the 2HDM and MSSM models. By the end of Run 2, we will be looking into four times larger dataset, hence should double our statistical sensitivity.

\section{Acknowledgements}

This work was partially supported by the Ministry of Science and Higher Education grant no. DIR/WK/2016/13.

\section{References}

[1] ATLAS Collaboration, Phys. Lett. B 716 (2012) 1. 
[2] CMS Collaboration, Phys. Lett. B 716 (2012) 30.

[3] ATLAS and CMS Collaborations, Phys. Rev. Lett. 114 (2015) 191803.

[4] ATLAS Collaboration, ATLAS-CONF-2017-047, https://cds.cern. ch/record/2273854.

[5] ATLAS and CMS Collaborations, JHEP 08 (2016) 045.

[6] A. Hill and J. van der Bij, Phys. Rev. D 36 (1987) 3463;

G. M. Pruna and T. Robens, Phys. Rev. D 88 (2013) 115012.

[7] T. D. Lee, Phys. Rev. D 8 (1973) 1226-1239;

J. F. Gunion and H. E. Haber, Phys. Rev. D 67 (2003) 075019;

G. Branco et al., Phys. Rept. 516 (2012) 1.

[8] G. Senjanovic and R. N. Mohapatra, Phys. Rev. D12 (1975) 1502.

[9] J. Gunion, R Vega and J Wudka, Phys. Rev. D42 (1990) 1673.

[10] H. Georgi and M. Machacek, Nucl. Phys. B262 (1985) 463.

[11] K. Agashe, R. Contino and A. Pomarol, Nucl. Phys. B 719 (2005) 165;

R. Contino, L. Da Rold and A. Pomarol, Phys. Rev. D 75 (2007) 055014.

[12] ATLAS Collaboration, JINST 3 (2008) S08003.

[13] ATLAS Collaboration, JHEP 09 (2016) 001.

[14] CMS Collaboration, Phys. Rev. Lett. 117 (2016) 051802.

[15] ATLAS Collaboration, arXiv:1707.04147 [hep-ex].

[16] ATLAS collaboration, J. High Energ. Phys. (2017) 2017: 112.

[17] ATLAS Collaboration, ATLAS-CONF-2017-058, https://cds.cern.ch/record/2273874.

[18] ATLAS Collaboration, arXiv:1708.09638 [hep-ex].

[19] ATLAS Collaboration, ATLAS-CONF-2017-055, https://cds.cern.ch/record/2273871.

[20] ATLAS Collaboration, arXiv:1710.07235 [hep-ex].

[21] ATLAS Collaboration, arXiv:1709.07242 [hep-ex].

[22] A. Djouadi, L. Maiani, G. Moreau, A. Polosa, J. Quevillon and V. Riquer, Eur. Phys. J. C73 (2013) 2650;

A. Djouadi, L. Maiani, G. Moreau, A. Polosa, J. Quevillon and V. Riquer, JHEP 1506 (2015) 168. [23] ATLAS Collaboration, Eur. Phys. J. C 76 (2016) 585.

[24] M. Drees, R. Godbole and P. Roy, Cambridge U. Press, 2006;

S. Martin, hep-ph/9709356.

[25] J. Gunion, H. Haber, G. Kane and S. Dawson, The Higgs Hunter's Guide, Reading 1990;

M. Spira, Fortschr. Phys. 46 (1998) 203;

A. Djouadi, Phys. Rept. 457 (2008) 1.

[26] ATLAS Collaboration, ATLAS-CONF-2016-088, https://cds.cern.ch/record/2206282.

[27] M. Carena et al., Eur. Phys. J. C 73 (2013) 2552.

[28] ATLAS Collaboration, Phys. Lett. B 759 (2016) 555.

[29] ATLAS Collaboration, ATLAS-CONF-2016-089, https://cds.cern.ch/record/2206809.

[30] ATLAS Collaboration, arXiv:1710.09748 [hep-ex].

[31] K. Huitu, J Maalampi, A Pietiläand M Raidal, Nucl. Phys. B 487 (1997) 27. 